\title{
Le " moment 68 " au Collège Sainte-Anne : la mentalité estudiantine au moment de la grève de 1968
}

\author{
Michael Poplyansky \\ Cité universitaire francophone de I'Université de Regina

\section{En collaboration avec Mylène Comeau'} \\ Université Sainte-Anne
}

\section{RÉSUMÉ}

À l'instar d'autres institutions postsecondaires de l'époque, le Collège Sainte-Anne du village acadien de Pointe-de-l'Église (Nouvelle-Écosse) vit aussi une grève étudiante en 1968. L'évènement déclencheur est un éventuel déménagement de l'institution à Yarmouth. Le présent article analyse la mentalité des étudiants de ce collège classique au moment de la grève. Il vise ainsi à combler, au moins partiellement, une lacune dans l'historiographie actuelle consacrée aux années soixante, qui est encore largement limitée aux centres urbains. L'article conclut que, sans être coupés des bouleversements qui frappent l'Occident dans la foulée de l'année 1968, les étudiants de Sainte-Anne maintiennent une certaine sensibilité conservatrice, ne cherchant pas à rompre brutalement avec leurs aînés.

\section{ABSTRACT}

Like other postsecondary institutions of the era, College Sainte-Anne, situated in the Acadian village of Church Point (Nova Scotia), experienced a student strike in 1968. The catalyst was the College's possible move to Yarmouth. The article analyses this classical college's students' mentality at the time of the strike. It seeks to fill, at least partially, a gap in "sixties" historiography, which is still largely focused on urban centres. The article concludes that, while not cut off from the upheaval that affected the entire Western world in the years following 1968, Sainte-Anne students maintained a conservative sensibility, refusing a brutal break with their elders.

1 Mylène Comeau, alors étudiante à l'Université Sainte-Anne et native de la baie Sainte-Marie, a fait bénévolement une partie importante de la recherche en vue de la rédaction de cet article, notamment au niveau de l'histoire orale. 
À la fin des années 1960 et au début des années 1970, le monde occidental dans son ensemble traverse une période de grande turbulence. À l'aube de leur âge adulte, les premiers baby-boomers, nés au début de l'après-guerre, rejettent, de multiples façons, le monde de leurs parents. Cette contestation se fait sentir notamment sur les campus universitaires. Que ce soit par le biais de la contreculture, ou de mouvements politiques de gauche, les étudiants appartenant à cette génération, dite "lyrique ", ${ }^{1}$ rêvent du début " d'un temps nouveau ». ${ }^{2}$

Les Maritimes ont aussi vécu leur " moment 68 ». ${ }^{3}$ Même si elles sont loin d'être représentatives de la totalité de l'opinion étudiante, nombreuses sont les luttes menées sur les campus universitaires à la fin des années 1960 et au début des années 1970. ${ }^{4}$ À l'Université de Moncton, la grève étudiante de février 1968 et l'occupation du pavillon des sciences l'année suivante, toutes deux provoquées par le manque de financement gouvernemental accordé à l'institution, attirent, depuis un certain temps déjà, beaucoup d'attention de la part des chercheurs. ${ }^{5}$ À la University of New Brunswick, des manifestations contre les cartes d'identité obligatoires, menées par un professeur antiguerre, Norman Strax, et ses alliés étudiants, se terminent par la suspension de Strax et une occupation infructueuse de son bureau. ${ }^{6}$ En Nouvelle-Écosse, les étudiants du King's College de l'Université Dalhousie s'activent au sein de l'aile jeunesse du Nouveau parti démocratique, luttant pour la syndicalisation de pêcheurs à Canso. ${ }^{7}$ Même à St. Francis Xavier University, de tradition catholique, des articles saluant la révolution sexuelle paraissent dans le journal étudiant. Les étudiants organisent aussi des "Teach-In" traitant de l'oppression des Noirs et des Autochtones, ainsi que de l'usage récréatif des drogues. ${ }^{8}$

Le Collège Sainte-Anne de Pointe-de-l'Église, collège classique géré par les pères eudistes depuis sa fondation en 1890, connaît également une grève étudiante en décembre 1968. L'élément déclencheur est un éventuel déménagement du collège à Yarmouth, une ville anglophone d'environ sept mille habitants, située à soixante kilomètres au sud-ouest du village de Pointe-de-l'Église. Un des principaux arguments évoqués contre le déménagement est donc la crainte que le collège ne perde son caractère francophone s'il devait désormais se situer dans une ville à grande majorité anglaise. ${ }^{9}$

La grève de Sainte-Anne est pourtant passée sous silence dans l'historiographie. L'histoire de l'Acadie contemporaine, surtout en ce qui a trait à l'éducation postsecondaire, est encore largement focalisée sur Moncton, et dans une moindre mesure, le nord du Nouveau-Brunswick. ${ }^{10}$ Ainsi, les expériences des Acadiens de la NouvelleÉcosse, même pendant des périodes charnières comme la fin des années 1960, demeurent encore passablement négligées.

\section{Problématique}

En se penchant sur la mentalité (ou l'ethos ${ }^{11}$ ) des étudiants du collège au moment de la grève, le présent article vise donc à combler ce vide, au moins partiellement. À la surface, il semblerait qu'avec leur grève, les étudiants de Sainte-Anne, malgré leur éducation religieuse et leur environnement isolé, s'inscrivent de plain-pied dans 
l'effervescence de la fin des années 1960. Pourtant, nous concluons que, malgré leur action contestataire, ils ne cherchent pas à s'opposer de manière frontale à leurs aînés. Les marqueurs séparant les générations y sont moins évidents qu'ailleurs. Cet article peut donc servir à nuancer les travaux classiques analysant le parcours de "la génération lyrique ". Pour l'essayiste François Ricard, par exemple, les premiers babyboomers nés entre les dernières années de la Seconde Guerre mondiale et le début des années cinquante partagent, grâce à la force du nombre, une série d'expériences collectives leur donnant une très forte identité générationnelle. Selon Ricard, " la génération a [...] le caractère d'une détermination lourde ", non seulement liant l'individu " à tel groupe d'âge ", mais lui « offrant un point d'ancrage dans le courant de l'histoire ". ${ }^{12}$ Même si les facteurs qui y contribuent dépassent le simple choc générationnel, ${ }^{13}$ les contestations dans la foulée de l'année 1968, entre les premiers baby-boomers et leurs aînés, (ou le " moment 68 ") sont au cœur de cette expérience historique partagée. Pourtant, le présent article se veut un rappel que le " moment 68 » se vit fort différemment selon l'espace géographique dont il est question.

Précisons d'emblée que, malgré leur grève, les étudiants de Sainte-Anne ne sont pas unanimement contre le déménagement à Yarmouth. Les avantages économiques du nouvel emplacement priment pour certains d'entre eux, surtout pour ceux qui sont originaires de la municipalité d'Argyle, en proximité de Yarmouth. ${ }^{14}$ D'autres étudiants sont inquiets de " perdre leur semestre " à cause de la grève. ${ }^{15}$ Pourtant, il est très difficile de trouver l'expression de ce point de vue dissident dans des sources documentaires de l'époque. Comme sur d'autres campus universitaires, la « minorité silencieuse " n'arrive pas à laisser une marque à Sainte-Anne à la fin des années $1960 .^{16}$

Ainsi, ce sont les étudiants qui choisissent d'appuyer activement la grève de décembre 1968 qui nous intéressent. Dans quelle mesure la contestation politique et culturelle associée à la fin des années 1960 imprègne-t-elle les mentalités de ces individus qui fréquentent un collège confessionnel situé dans le milieu rural et géographiquement isolé qu'est la région de Clare? ${ }^{17}$

En tant qu'étude de cas, le Collège Sainte-Anne est particulièrement intéressant étant donné la composition de son corps étudiant. En 1968-1969, parmi ses 174 étudiants, Sainte-Anne compte huit anglophones (qui suivent tous leurs cours en anglais), ainsi que des Acadiens. ${ }^{18}$ Cela distingue Sainte-Anne d'autres collèges classiques francophones de la fin des années 1960. Il y a aussi une trentaine d'étudiants québécois, surtout des Madelinots. ${ }^{19}$ Une solidarité étudiante est quand même maintenue à Sainte-Anne, malgré les différences ethnolinguistiques. Jusqu'à quel point ces individus agissent-ils donc avec « les tendances sociales et culturelles balayant tous les continents "? ${ }^{20}$

Dans une étude récente devenue un ouvrage de référence en études acadiennes, "Le moment 68 " et la réinvention de l'Acadie, l'historien Joel Belliveau conclut que l'action des étudiants de l'Université de Moncton se déroule en synchronie avec celle de leurs collègues ailleurs en Occident. À partir de la fin des années 1960, tous deviennent plus radicaux dans leurs revendications, que ce soit en adhérant à la culture des drogues, ou à travers de mouvements politiques, tels le maoïsme, l'anarchisme, 
ou le tiers-mondisme. ${ }^{21}$ À cause de la situation démo-linguistique du sud-est du Nouveau-Brunswick, la révolte des étudiants de Moncton s'exprime à travers une adhésion à ce que Belliveau appelle le " communautarisme ", où ils réclament des " des droits collectifs et un traitement particulier pour la communauté francophone ». ${ }^{22}$ Les évènements de Moncton seraient donc la manifestation locale d'un phénomène global. Il s'agit d'une interprétation très convaincante. Pourtant, les mêmes constats peuvent-ils réellement s'appliquer au Collège Sainte-Anne? De quelle manière, les étudiants de Sainte-Anne, qui se trouvent forcément dans un contexte très différent de celui de Moncton, apprivoisent-ils le climat mondial contestataire dans lequel ils se trouvent?

\section{Méthode}

Pour répondre à cette question, nous avons mené un dépouillement systématique du journal étudiant Le Reflet pour les années 1967-1968 et 1968-1969. ${ }^{23}$ En tant que journal mensuel ${ }^{24}$ bilingue, Le Reflet fournit une tribune de choix à ceux qui sont les plus impliqués dans la grève de 1968, tant anglophones que francophones. Signe de la transformation des collèges classiques à cette période, Le Reflet n'a plus de prêtres " aviseurs " dans son comité de rédaction à partir de novembre 1967. ${ }^{25}$ Les étudiants n'hésitent donc pas à publier des articles qui déplaisent aux pères eudistes. ${ }^{26}$ Nous pouvons donc présumer que le journal reflète bien la pensée de ceux et celles qui y écrivent. Ainsi, le journal, davantage que les archives du Collège Sainte-Anne dont le contenu est essentiellement administratif, semble être la meilleure voie pour saisir la mentalité du corps étudiant du collège. ${ }^{27}$

Afin de mieux comprendre l'interaction entre les étudiants et la communauté dont ils sont issus, nous avons procédé à un dépouillement ponctuel des journaux locaux, dont le Petit Courrier, le Yarmouth Vanguard, le Digby Courier, et le Halifax Chronicle Herald. Nous avons aussi consulté le dossier de presse consacré à la grève étudiante, conservé dans les archives de l'Université Sainte-Anne. Enfin, pour supplémenter les sources documentaires, nous avons mené une enquête orale en 2015, auprès de sept des étudiants impliqués dans la grève. ${ }^{28}$ Bien que l'histoire orale demeure, pour certains, une méthode contentieuse, ${ }^{29}$ il nous a paru souhaitable d'interviewer ceux et celles qui avaient vécu personnellement les évènements dont il est question dans cet article. À cet égard, nous nous sommes inspirés d'une série de travaux en histoire du Canada, qui témoignent d' " une tendance au croisement de l'oral et de l'écrit, les premières sources souvent utilisées en complément à des sources écrites lacunaires pour traiter d'enjeux historiques du temps présent $"{ }^{30}$ Dans notre cas, les témoignages oraux se sont révélés facilement conciliables avec les documents d'archives.

\section{Historiographie}

Ultimement, l'article représente une étude de cas de la manière dont un phénomène global, soit la contestation politique et culturelle de la fin des années 1960 et du 
début des années 1970, affecte les étudiants dans les collèges classiques en Acadie. Cette question n'occupe actuellement pas beaucoup de place dans l'historiographie. En fait, la mentalité des étudiants n’est pas nécessairement au cour des histoires institutionnelles consacrées à ces établissements.

Procédons donc à un examen de ces ouvrages, dans la mesure où ils traitent de la pensée et de l'action étudiante. Construire un savoir : l'enseignement supérieur au Madawaska 1946-1974 de Jacques Paul Couturier, relate, en quelques pages, les perturbations qui touchent les collèges Saint-Louis et Maillet au tournant des années 1970. Mais malgré l'opposition des étudiants des collèges Saint-Louis et Maillet à plusieurs règlementations institutionnelles, ils demeurent, " timides en regard des manifestations [...] qui se déroulent [...] ailleurs en Occident ", n'intervenant pas dans les grands débats politiques et culturels de l'heure. ${ }^{31}$ Dans son étude de l'enseignement supérieur à Shippagan, Nicolas Landry arrive à des conclusions similaires. Se concentrant surtout sur leur journal étudiant, Le Stella Maris, il démontre que les jeunes filles qui fréquentent le Collège Jésus-Marie dans les années 1960 ne se révoltent pas contre le monde qui les a vues naître. Par exemple, elles valorisent le rôle de mère et d'épouse — minimisant l'importance de l'apprentissage du grec pour une femme au foyer - et rejettent fermement la musique " rock ». ${ }^{32}$ Malheureusement, cette analyse du Stella Maris est surtout axée sur le début des années 1960 et ne permet pas de savoir jusqu'à quel point la situation évolue vers la fin de la décennie.

Il y a un peu plus de turbulence au collège de Bathurst pendant les années 1960. Cela ressort d'une autre monographie de Nicolas Landry : Un collège classique au Nouveau-Brunswick : le Sacré-Cour de Caraquet-Bathurst, 1899-1975. ${ }^{33}$ À la différence des étudiants du Madawaska, ${ }^{34}$ les étudiants de Bathurst contemplent faire la grève pour appuyer leurs confrères de Moncton en février $1968 .{ }^{35}$ Landry y relève aussi les grandes tensions entre étudiants et administrateurs, surtout au moment où ces derniers s'apprêtent à transformer l'institution en collège communautaire au début des années $1970 .{ }^{36}$ Il constate également l'implication des étudiants dans les causes hors campus, notamment celles du développement régional et des droits linguistiques. ${ }^{37}$ Toutefois, comme Landry l'admet lui-même, son étude est avant tout " une biographie institutionnelle », ce qui fait en sorte que la mentalité des étudiants ne peut y être analysée en profondeur. ${ }^{38}$

Il reste donc beaucoup à faire pour comprendre la pensée et l'action des étudiants des collèges classiques en Acadie à la fin des années 1960 et au début des années 1970. Cela est d'autant plus vrai dans le cas du Collège Sainte-Anne. Même si nous reconnaissons la grande valeur de Sainte-Anne, collège et université 1890-1990 ${ }^{39} \mathrm{de}$ René LeBlanc et Micheline Laliberté, la parole y est assez rarement donnée aux étudiants, surtout dans la période de l'après 1960. À part l'observation que les étudiants de Sainte-Anne font cause commune avec l'administration du collège pour maintenir l'institution à Pointe-de-l'Église, LeBlanc et Laliberté n'expliquent pas comment les étudiants conceptualisent leur action par rapport à l'esprit de contestation qui frappe alors le monde occidental. 
Dans le présent article, nous concluons qu’à la fin des années 1960, les étudiants de Sainte-Anne ne sont pas coupés des débats sociopolitiques, qui se déroulent à l'extérieur de la baie Sainte-Marie, que ce soit en Nouvelle-Écosse, au Canada, ou dans le monde occidental en général. Par exemple, les étudiants se mobilisent pour un meilleur financement postsecondaire et contre la discrimination raciale; dans certains cas, ils manifestent également de l'ouverture face au mouvement indépendantiste québécois. ${ }^{40}$ Pourtant, ils restent profondément hostiles à la confrontation avec leurs aînés. Même si certains adoptent un discours agressif dans la défense de leurs idées pendant leur grève, et même s'ils cherchent à se solidariser, au moins jusqu'à un certain point, avec des regroupements contestataires, comme le Black United Front en Nouvelle-Écosse ou le Mouvement pour l'intégration scolaire au Québec, ${ }^{41}$ ils demeurent largement étrangers à la contreculture et aux idéologies de gauche, communément associées à la fin des années 1960 et au début des années 1970 (période qu'on appelle aussi la fin des "sixties»). Ces tendances se font sentir ailleurs dans les Maritimes, mais elles demeurent en grande partie absentes du Collège Sainte-Anne.

Pour en faire la démonstration, nous ferons un survol de la manière dont les « sixties " ont été vécues au Canada, avec une en mettant l'accent sur sur les institutions d'enseignement postsecondaire des Maritimes. Nous nous tournerons alors vers la communauté de Clare et le Collège Sainte-Anne. D'abord, nous présenterons les changements modernisateurs qui y atteignent de nouveaux sommets dans les années 1960, ainsi que les évènements qui mènent directement à la confrontation de décembre 1968. Deuxièmement, nous montrerons que, même si des changements similaires peuvent pousser certains étudiants à faire des revendications encore plus radicales ailleurs, ce n'est pas le cas à Sainte-Anne. La sensibilité conservatrice des étudiants se poursuit jusqu'à la fin des années 1960. Enfin, dans la dernière partie du texte, traitant spécifiquement de l'action des étudiants pour garder Sainte-Anne à Pointe-de-l'Église, nous soutiendrons que même s'ils empruntent certains discours et tactiques qui ont cours ailleurs, cela ne reflète pas nécessairement un rejet global du mode de vie de leurs aînés.

\section{L'esprit des « sixties »}

Les "sixties" ne correspondent pas à la décennie des années 1960. Au Canada, certains historiens définissent la période comme allant de 1959-date de la première manifestation contre les armements nucléaires menée par la Combined Universities Campagin for Nuclear Disarmament ${ }^{42}$ —à 1973, année du premier choc pétrolier. ${ }^{43}$ Il s'agit d'une période de grande prospérité. Au Canada, par exemple, le taux de croissance économique se situe entre $4 \%$ et $6,5 \%$ et le taux de chômage se maintient dans une situation de plein emploi entre 1965 et $1972 .{ }^{44}$

Malgré cette continuité au niveau économique, les historiens "des sixties» ont tendance à diviser cette période en deux grandes phases. ${ }^{45}$ La première, allant jusqu'en 1968, représente une période assez "bon-enfant ". Les institutions postsecondaires, poursuivent leur l'évolution, qui date de la fin de la Seconde Guerre mondiale. Politiciens et intellectuels mettent en valeur une éducation supérieure accessible à 
tous, comme un élément clé de la primauté occidentale en période de guerre froide. ${ }^{46}$ Les femmes, jadis largement exclues du monde universitaire, y sont de plus en plus présentes, la prospérité économique aidant à ce que leur éducation ne soit plus autant considérée comme une menace. ${ }^{47}$ Par conséquent, une fois que les premiers babyboomers arrivent à l'âge adulte, les inscriptions universitaires montent en flèche, le nombre d'étudiants augmentant de $192 \%$ au Canada entre 1960 et $1969 .{ }^{48}$

Parallèlement aux changements vécus dans le milieu universitaire, les collèges classiques francophones évoluent aussi après la Seconde Guerre mondiale. ${ }^{49}$ Ils "perdent leurs défauts les plus évidents : lourdeur, passéisme, intolérance, élitisme "; les méthodes pédagogiques "se mettent lentement à jour "; les livres, peut-être toujours à l'index, sont plus facilement accessibles; les infrastructures physiques se renouvellent également. ${ }^{50}$ Dans certains collèges, la laïcisation progressive du corps professoral entraîne l'introduction d'idées non conventionnelles, notamment en ce qui a trait au rapport entre les genres. Désormais, ces innovations ne sont pas nécessairement rejetées par tous les responsables religieux. ${ }^{51}$ Les étudiants des collèges, quant à eux, commencent aussi à se percevoir comme " une catégorie sociale autonome ". ${ }^{52}$ En Acadie, par exemple, le 24 novembre 1963, les représentants des six collèges classiques des Maritimes se rencontrent à Moncton pour fonder l'Union générale des étudiants acadiens. Elle devient la Fédération des associations générales étudiantes des collèges acadiens en $1964 .^{53}$ En contrepartie, comme le note François Ricard, les changements «ne privent pas les collèges de leurs qualités les plus précieuses : taille relativement modeste des établissements, encadrement des étudiants, importance accordée à la formation générale, sens de la culture ". ${ }^{54}$

Ainsi, l'esprit des étudiants au début des « sixties » est assez optimiste. Cet optimisme est renforcé par l'expansion de l'État. Dans son étude du mouvement étudiant québécois, Jean-Philippe Warren fait un constat qui vaut autant pour les Maritimes : au début des années soixante, les étudiants, que ce soit dans les universités ou dans les collèges, voient encore l'État comme un levier de développement. ${ }^{55}$ Comme au Québec, l'État provincial dans les Maritimes est alors en pleine expansion. Parmi les nouvelles incitatives, l'on retrouve la consolidation des écoles, le financement provincial, plutôt que local, des services sociaux, des subventions directes aux universités, et de nouvelles bourses d'études. ${ }^{56}$ Sans que ce soit nécessairement explicite, ces réformes permettent à l'État de mieux servir les groupes historiquement désavantagés, dont les Acadiens. ${ }^{57}$

Pourtant, les parties rurales des Maritimes, bien souvent des régions à forte concentration acadienne, demeurent économiquement pauvres. Il y subsiste des tensions raciales qui sont particulièrement mises en évidence lors de l'épisode d'Africville, et la dégradation environnementale devient un problème de plus en plus grave. ${ }^{58}$ Une désillusion similaire se vit ailleurs en Occident. ${ }^{59}$

À la fin des années 1960, s'amorce donc la deuxième phase des "sixties ", où certains parmi les premiers nés du baby-boom commencent à se révolter de manière marquée. L'on constate l'émergence d'une culture alternative (contreculture), offrant " une autre façon de vivre, de voir le monde ou de se lier aux êtres ". ${ }^{60}$ Cette contreculture ne se limite pas aux plus grandes villes. À la fin des années 1960, les enquêteurs 
de la Gendarmerie royale (GRC) sont également présents sur le campus de St. Francis Xavier. Comme ailleurs au Canada, ils cherchent, à travers leur observation des étudiants, à définir la catégorie sociale de " hippie " et à l'utiliser comme un repoussoir dans la bataille publique de la GRC contre la légalisation de la marijuana. ${ }^{61}$

L'on assiste également au déploiement de mouvements politiques, clairement marqués à gauche. Au Québec et en Acadie du Nouveau-Brunswick, comme dans d'autres régions périphériques de l'Occident, le processus de décolonisation inspire un renouveau du mouvement nationaliste. ${ }^{62}$ Du côté anglophone, le mouvement du "Waffle " dépasse ses origines ontariennes et s'implante dans les Maritimes. ${ }^{63}$ Ainsi, à partir du moment où il devient évident que les grandes promesses de l'après-guerre ne se sont pas réalisées, de nombreux étudiants des Maritimes, tant anglophones que francophones, commencent à adhérer à des idéologies marquant la rupture avec les consensus sociaux de leur époque.

Jusqu'à maintenant, toutefois, l'historiographie traitant de la fin des " sixties " au Canada, et même dans les Maritimes, est encore concentrée sur des régions urbaines. Tel que nous l'avons mentionné, les histoires institutionnelles des collèges classiques acadiens, en dépit de leurs grandes qualités, ne nous fournissent qu'un portrait limité de la mentalité étudiante pendant cette période. Se pourrait-il que dans certains collèges classiques, comme Sainte-Anne, malgré un processus de modernisation bien enclenché, les jeunes maintiennent une plus grande solidarité avec les aînés, au moment précis où leurs confrères en milieu urbain se lancent dans un processus de rupture?

\section{La transformation du Collège Sainte-Anne dans les années 1960}

Bien que la baie Sainte-Marie n'accède pas à la modernité à l'aube des années 1960 — selon la sociologue Michelle Landry, l'Acadie était " moderne " dès ses débuts dans le sens qu'elle est née d'un " processus d'accroissement des contacts, de communications et d'échanges entre différents peuples " ${ }^{64}$ - elle vit un processus de modernisation socio-économique, similaire à celui qui touche d'autres parties du Canada. ${ }^{65}$ Dans la période de l'après-guerre, cela se traduit par l'expansion de l'État et le développement d'une technocratie de plus en plus puissante. Sans perdre les caractéristiques qui distinguent la baie Sainte-Marie des régions plus urbaines, ${ }^{66}$ la communauté est touchée par ces mêmes phénomènes.

Ce processus est bien décrit dans les travaux de l'anthropologue Marc-Adélard Tremblay. ${ }^{67}$ Certes, après la Seconde Guerre mondiale, les résidents doivent surmonter des défis : déclin des chantiers navals, transformation de l'industrie de la pêche, émigration persistante vers les grands centres. ${ }^{68}$ À cause de ces départs, la population de Clare, environ neuf mille habitants sur une superficie de $852,82 \mathrm{~km}^{2}$, reste pratiquement inchangée depuis $1910 .{ }^{69}$ En même temps, les réformes étatiques de l'après-guerre affectent aussi la population de Clare. Malgré quelques réticences, la consolidation des écoles est finalement saluée par la plupart des habitants. ${ }^{70}$ Par ailleurs, avec l'expansion de l'État, " il faut des organisations spécialisées, de nouveaux canaux de communication pour exercer des pressions auprès des gouvernements afin de promouvoir des législations nouvelles favorables à la minorité acadienne $» .{ }^{71}$ Les 
possibilités de mobilité sociale deviennent aussi plus grandes; en plus des professions traditionnelles de médecin, dentiste ou avocat, de nouveaux postes administratifs (dans le secteur public ou privé) peuvent offrir des débouchés intéressants aux habitants de la région. ${ }^{72}$

Au Collège Sainte-Anne proprement dit, il y a aussi des transformations. En 1961, l'institution ouvre ses portes aux femmes. Les pratiques disciplinaires s'assouplissent et le curriculum se met à jour. Le grec n'est plus au programme depuis le début des années 1960. Puis, en 1962-1963 le collège "introduit un cours classique restructuré "; pour les trois dernières années du cours universitaire, les étudiants peuvent " choisir un certain nombre de cours à option, répartis en trois groupes : Lettres, Sciences, Sciences de l'Homme $"{ }^{73}$ À partir de 1960, un plus grand nombre de laïcs se joignent également au corps professoral. ${ }^{74}$ Une association de professeurs est fondée en $1967 . .^{75}$ S'il y a toujours des prêtres " aviseurs " dans l'équipe de rédaction du journal étudiant, ces derniers perdent de l'influence. ${ }^{76}$ Face à cette ouverture, les étudiants exigent des réformes supplémentaires, qu'ils obtiennent sans trop de difficulté. ${ }^{77}$ En 1967, Le Reflet acquiert sa pleine autonomie du corps professoral, tout comme l'Association générale des étudiants du Collège Sainte-Anne (AGECSA). ${ }^{78}$ Fondée cinq ans auparavant, et déjà affiliée à la Fédération acadienne générale des étudiants des collèges acadiens, l'AGECSA participe aussi, en 1967, à la création de la Nova Scotia Union of Students. ${ }^{79}$ Son premier objectif est d'améliorer le soutien financier accordé aux étudiants du postsecondaire. ${ }^{80}$ Ainsi, le Collège Sainte-Anne se " dépoussière ", tout en gardant ses meilleures qualités : son environnement intime, sa rigueur pédagogique, ses activités parascolaires. ${ }^{81}$

Pourtant, il y a aussi le « revers de la médaille ». Au tournant des années 1960, vu le manque de vocations chez les eudistes, les pères commencent à envisager leur retrait du collège ${ }^{82}$. En 1966, avec l'avenir de l'institution remis en cause, Mgr Jacques Garneau, directeur de l'Association des universités et collèges du Canada, publie un rapport pour le Comité des subventions aux universités et collèges du gouvernement de la Nouvelle-Écosse (Grants Committee), recommandant le déménagement du Collège Sainte-Anne à Yarmouth. D'après Mgr Garneau, un nouvel emplacement permettrait au collège d'attirer davantage de professeurs et d'étudiants, tant anglophones que francophones ${ }^{83}$.

Une fois le rapport Garneau déposé, l'élite acadienne de Clare répond par un discours très diplomate. Au milieu des années 1960, cette élite composée de clercs, de professionnels, de commerçants et d'administrateurs se charge du maintien de la nationalité acadienne. L'élite accepte les prémisses de la modernisation : la survie de la nationalité dépend d'un rehaussement du niveau de vie et d'une plus grande scolarisation. ${ }^{84}$ Pour cette élite nationaliste, il est également primordial de maintenir la bonne entente avec la majorité anglaise, qui représente $95 \%$ de la population néo-écossaise, ainsi que $66 \%$ des résidents des comtés de Yarmouth et Digby. ${ }^{85}$ L'on refuse donc de polariser l'opinion publique sur des bases linguistiques. Lors d'un rassemblement tenu suite au dépôt du rapport Garneau, attirant environ cinq cents personnes, V. F. Melanson, manager du "Digby County Power Board ", met l'emphase sur les avantages économiques que procure la présence du Collège Sainte-Anne 
dans Clare. Son confrère, Julius Comeau, un dentiste de Meteghan, est encore plus explicite en évoquant la bonne entente entre communautés linguistiques : "We peace loving Acadians are glad to share our culture with others and we are also glad to be able to share the culture of others ", déclare-t-il. Comeau termine son discours avec le souhait que les délibérations concernant l'avenir du Collège Sainte-Anne soient définies par " the good relationship, the good entente between gentlemen of different language and culture ". ${ }^{86}$ En septembre 1967, cette volonté de bonne entente est concrétisée lorsque, pour "mieux s'adapter aux besoins locaux ", notamment ceux des anglophones de Clare $^{87}$ et des communautés avoisinantes, l'administration du collège décide d'accueillir des étudiants d'origine anglaise, sans qu'ils aient à suivre un cheminement en français. ${ }^{88}$

En mai 1968, le Grants Committee donne enfin suite au rapport de Mgr Garneau en recommandant officiellement au gouvernement provincial le déménagement du Collège Sainte-Anne à Yarmouth. Le premier ministre néo-écossais, George Smith, quant à lui, indique que "le gouvernement n'accepte pas a priori ces recommandations " et s'engage à étudier sérieusement les "valeurs culturelles attachées au Collège Sainte-Anne $"{ }^{89}$ L'on se trouve dans une période d'incertitude; les résidents de Clare, y compris les membres de l'administration du collège, se mobilisent donc pour influencer la prise de décision gouvernementale et garder Sainte-Anne à Pointe-de-l'Église. ${ }^{90}$

Parmi ceux qui s'activent, il y a bien sûr les étudiants de Sainte-Anne. Le 5 décembre 1968, suite à la décision du diocèse de Yarmouth d'appuyer le déménagement, quatre-vingt-neuf pour cent des cent soixante-quatorze étudiants votent une déclaration de grève. ${ }^{91}$ Les étudiants de Sainte-Anne ont un soutien indéfectible de la part de l'association des professeurs. ${ }^{92}$ Celle-ci affiche clairement son appui à la grève et condamne la décision du diocèse d'appuyer le déménagement à Yarmouth, y voyant une menace aux rapports harmonieux entre paroissiens du sud-ouest de la Nouvelle-Écosse. ${ }^{93}$ Le 13 décembre, la session est écourtée; neuf jours plus tard, les étudiants acceptent de revenir en classe après les vacances de Noël. Afin de dénouer la crise, le gouvernement provincial s'est engagé à nommer une commission d'enquête, présidée par David Munroe, adjoint du recteur de l'Université McGill, pour étudier la question du Collège Sainte-Anne. ${ }^{94}$ Ultimement, le collège va demeurer sur son site original, où il se trouve encore aujourd'hui. ${ }^{95}$

Au-delà de cette histoire évènementielle, la grève à Sainte-Anne est révélatrice, car les étudiants, contrairement à ceux de Moncton, par exemple, ${ }^{96}$ n'en profitent pas pour faire avancer des idéologies alternatives qui dépassent la simple question de l'emplacement du collège. Il s'agit plutôt d'un mouvement de solidarité communautaire, auquel les jeunes participent. En effet, à l'époque de la grève, les étudiants de Sainte-Anne se distinguent par leur conservatisme.

\section{La sensibilité conservatrice des étudiants}

Comme le soulignent les politologues Danic et Ian Parenteau, le conservatisme ne repose pas nécessairement sur un vaste corpus théorique. Reprenant Michael 
Oakeshott, ils nous rappellent qu'il s'agit plutôt d'une disposition d'esprit. ${ }^{97}$ Ils identifient néanmoins plusieurs idées maîtresses du conservatisme, dont le respect de la tradition, de la hiérarchie et de la cohésion sociale, ainsi qu'un certain moralisme. ${ }^{98}$ Tous ces principes se retrouvent dans la pensée des étudiants de Sainte-Anne à la fin des années 1960.

Le contexte dans lequel Sainte-Anne vit son « moment 68 » est donc très différent de celui qui prédomine dans d'autres localités, privilégiées par les historiens qui se penchent sur les années 1960 et 1970 . Cela se manifeste dans plusieurs domaines. Lorsque l'Association étudiante du Collège Sainte-Anne acquiert son autonomie par rapport au corps professoral en novembre 1967, elle promet de l'exercer sagement. Son président, Alain Deveau, écrivant dans Le Reflet rappelle que " plus que jamais, il nous faut être responsable; les Autorités nous prêtent confiance, à nous de respecter cette confiance. Désormais, avant d'agir il nous faut étudier les avantages et les désavantages que nos gestes peuvent apporter [...]. [I]l nous faut désormais agir en hommes responsables. " ${ }^{99}$ L'éditorialiste du Reflet, Jean-Robert Turcotte, rejette également la notion du "pouvoir étudiant ". Il affirme toujours le bienfondé d'une certaine déférence envers les aînés. Selon lui, l'étudiant doit d'abord « assurer sa formation académique et personnelle de manière à pouvoir donner à son jugement une capacité réelle de logique, de précision et de durabilité ". ${ }^{100}$ Il s'agit certainement d'un point de vue non négligeable. L'étudiante Madeleine Deveau salue aussi dans Le Reflet le manque relatif de conflit entre administrateurs et étudiants, ce qui distinguerait de façon positive le Collège Sainte-Anne par rapport aux autres institutions postsecondaires de l'époque, marqués par des «campus riots, protests and sit-ins». "Thankfully", écrit-elle, "St-Anne have [sic] not resorted to such drastic methods. Let's hope that we are mature enough to be treated as adults and the two generations at St-Anne will work peacefully together. " ${ }^{101}$

D'autres articles dans Le Reflet témoignent aussi d'une sensibilité conservatrice. Par exemple, l'étudiant Robert Gaudet critique les « mariages d'essai » avec un argumentaire on ne peut plus traditionaliste. "Le mariage est une institution sérieuse ", écrit-il, le mariage d'essai ne ferait que "détruire une valeur solidement établie : la famille " et serait fort nuisible pour les enfants qui en seraient le résultat. ${ }^{102} \mathrm{Un}$ autre article vante les vertus de la maternité; selon l'étudiante Anna Marie Thibodeau, « all women naturally aspire to attain the glory of motherhood. It is a feeling inborn in them and a potency waiting only for its moment of fulfillment. " ${ }^{103}$ Les propos de Thibodeau sont tirés d'une dissertation ayant remporté un "classroom essay competition " et le journal les publie sans commentaires ou critiques.

La sensibilité conservatrice des étudiants se manifeste aussi dans le domaine de la politique. Par exemple, dans Le Reflet, l'étudiant David Bourque, tout en admettant que " the youth of today, when he thinks of the world which he is going to inherit, becomes depressed and full of doubt ", vante tout de même les mérites de la guerre au Vietnam et les valeurs sûres que représentent le patriotisme et l'anticommunisme. ${ }^{104}$ Même si le point de vue de Bourque n'est peut-être pas unanimement partagé, il est quand même révélateur que le journal étudiant ne publie pas immédiatement de "réplique " à ses propos. ${ }^{105}$ 
Il ne semble pas y avoir non plus de véritable contreculture étudiante. En effet, lorsque, en 1968, les parents écrivent des lettres d'opinion aux journaux pour endosser le maintien du collège à Pointe-de-l'Église, ils le font en évoquant la préservation d'une culture qu'ils peuvent facilement partager. L'expérience collégiale à la baie Sainte-Marie implique la continuation des concerts des Jeunesses musicales et la reproduction des pièces de théâtre classiques; ce sont donc des activités qui ne font aucunement peur aux adultes et ne s'inscrivent pas du tout dans le stéréotype d'une époque marquée par le "sex, drugs and rock n'roll». ${ }^{106}$ Comme le conclut l'étudiante Gail Hersey "we were rural people; everything was low-key; there was nobody doing anything [...] illegal or at least I didn't know about it. It was a good atmosphere ${ }^{107}$

Il serait faux de prétendre que les étudiants sont totalement fermés au climat de la fin des années 1960. Certaines transformations sont peut-être plus facilement acceptables; par exemple, la même Madeleine Deveau qui salue l'absence de conflit avec ses professeurs évoque fièrement sa décision de porter une mini-jupe à l'université, malgré l'opposition de certains eudistes. ${ }^{108}$ " Je posais beaucoup de questions dans les classes de philosophie et de religion et ce n'était pas toujours une bonne chose ", ${ }^{109}$ se remémore-t-elle. D'autres étudiants se plaignent de la nourriture servie à la cafétéria ou de divers règlements administratifs. ${ }^{110}$ Mais lorsqu'il s'agit de participer aux grandes luttes politiques de la fin des années 1960 - décolonisation, féminisme, pacifisme, et ainsi de suite - les étudiants de Sainte-Anne, dans leur ensemble, sont largement absents. Les étudiants sont bel et bien conscients de ce qui se passe à l'extérieur de la baie Sainte-Marie, ce qui est évident dans leurs critiques des « mariages d'essai » ou des manifestations étudiantes ayant lieu sur d'autres campus universitaires. Ils prennent néanmoins la décision de rejeter de larges pans de la «culture jeune » de leur époque.

Même si trouver une explication définitive pour le conservatisme des étudiants de Sainte-Anne est difficile, plusieurs hypothèses méritent d'être soulevées. Il serait tentant de conclure que la réalité très minoritaire des Acadiens de la NouvelleÉcosse les rend moins prompts à opposer les figures d'autorité. ${ }^{111}$ Cependant, les étudiants anglophones de Sainte-Anne ne sont pas plus contestataires que leurs collègues francophones. L'attitude estudiantine semble plutôt refléter l'impossibilité d'une véritable rupture idéologique au sein d'une structure sociale communautaire (ou pour reprendre les mots du sociologue allemand Ferdinand Tönnies d'une " gemeinschaft ${ }^{112}$ ). À la baie Sainte-Marie, où les habitants se connaissent tous sur une base presque intime, il est difficile d'ouvrir sur un véritable pluralisme idéologique. Les universités des Maritimes où il y a le plus de contestation, comme l'Université Moncton et la University of New Brunswick, sont de plus grande taille que SainteAnne; leurs étudiants proviennent d'origines plus diverses et ont davantage de liens avec le mouvement étudiant pancanadien. ${ }^{113}$

Par ailleurs, à Moncton et à Fredericton, il y a des professeurs comme Roger Savoie, Jean-Paul Hautecoeur, Alain Even ou Norman Strax, qui ont une formation étrangère et qui partagent leurs idées novatrices avec les étudiants. ${ }^{114}$ Le collège classique acadien où il y a probablement le plus de contestation à la fin des années 1960 et au début des 1970, le Collège de Bathurst, a aussi, en son sein, des professeurs comme le futur chef du Parti acadien Euclide Chiasson, qui sont prêts, au moins 
jusqu'à un certain point, à aller à l'encontre des structures établies. ${ }^{115}$ Mais il ne semble pas que cette situation se répète au Collège Sainte-Anne. ${ }^{116}$ Les pères eudistes sont certes touchés par le renouveau du catholicisme. Par exemple, en 1968, ils permettent aux étudiants de participer à un colloque sur l'homme et la foi à Moncton où des intellectuels, comme le directeur du Devoir, Claude Ryan, influencés par le personnalisme, leur rappellent que la foi est censée être "une réponse libre à un appel ». ${ }^{117}$ Toutefois, de façon générale, les professeurs ne sont pas prêts à remettre en cause les fondements traditionnels du vivre ensemble : mariage, famille, déférence envers les figures d'autorité. Ainsi, pour les étudiants de Sainte-Anne, maintenir l'alliance avec les aînés de la communauté demeure relativement facile, même au moment où ils embarquent dans la grève de 1968.

\section{Garder Sainte-Anne à Pointe-de-l'Église : une contestation très relative}

Rappelons qu'un des principaux arguments pour le maintien de Sainte-Anne à Pointe-de-l'Église est son caractère francophone. Ainsi, certains étudiants font des emprunts aux discours indépendantistes qui ont cours au Québec. De prime abord, cela peut paraître comme un signe de rupture avec leurs aînés et avec la volonté de «bonne entente » que les Acadiens de la baie Sainte-Marie manifestent traditionnellement à l'endroit de leurs concitoyens anglophones. Pourtant, il s'agit d'un portrait assez incomplet de la situation.

Il est important de préciser que ce sont surtout les étudiants québécois qui s’inspirent de discours indépendantistes. Par exemple, dans une entrevue au journal Le Soleil, Jean-Pierre Vallée, étudiant originaire du Québec, mais vivant dans Clare depuis 1960, affirme même vouloir faire appel au Mouvement pour l'intégration scolaire (MIS) afin de maintenir Sainte-Anne à Pointe-de-l'Église. ${ }^{118}$ Formé de parents nationalistes, le MIS cherche à imposer l'éducation française aux immigrants qui viennent s'établir au Québec et est au cœur des affrontements de 1968 dans la banlieue montréalaise de Saint-Léonard. Quarante pour cent des habitants sont d'origine italienne, et cherchent, en grande majorité, à faire instruire leurs enfants en anglais. Grâce aux efforts du MIS, la commission scolaire locale proclame, en juin 1968, le français comme unique langue d'instruction. Par ailleurs, dans cette même entrevue au Soleil, Vallée affirme aussi vouloir chercher l'appui de René Lévesque, dans l'espoir que le leader indépendantiste se prononce en faveur du collège. ${ }^{119}$ Sans suggérer explicitement que le Collège Sainte-Anne devienne une institution unilingue française, un autre étudiant québécois, Denis Bradet, évoque également l'affaire Saint-Léonard dans Le Reflet, prétextant qu'à l'instar de cette crise québécoise "le problème du Collège risque de devenir un cas type » dans « l'éveil des consciences nationales [...] parmi les Acadiens du sud-ouest de la Nouvelle-Écosse ". ${ }^{120}$ Un troisième étudiant québécois, Mark Bradley, assure ses confrères de la baie Sainte-Marie que « dès maintenant, les Québécois seront prêts à vous aider en autant qu'ils en seront capables et en autant que vous aurez besoin d'eux ». Terminant son article dans Le Reflet avec un «Vive la France! Vive l'Acadie! Vive le Québec! », l'absence du «Vive le Canada! » est notée avec ironie par la rédaction. ${ }^{121}$ 
Les appels au MIS ou à René Lévesque n'aboutissent à rien. Les grévistes de Sainte-Anne doivent se contenter du soutien de diverses associations étudiantes dans les Maritimes, dont celles des universités et collèges néo-écossais Acadia, St. Mary's, King's et Mount St. Vincent. ${ }^{122}$ Par ailleurs, ils reçoivent aussi l'encouragement de l'Association étudiante du collège de Bathurst. Sous la présidence du futur militant du Parti acadien, Jean-Marie Nadeau, l'association envoie un télégramme au ministre néo-écossais de l'Éducation, Gérald Doucet, l'informant de l'appui de l'association pour " nos confrères étudiants de la Pointe-de-l'Église [sic] » et rappelant au ministre que "l'élément français est à considérer " dans toute prise de décision quant à l'avenir du collège. ${ }^{123}$

Il reste que personne à Sainte-Anne ne semble s'inspirer de la décolonisation comme le font de nombreux militants étudiants à la fin des années $1960 .{ }^{124}$ Même les étudiants québécois, qui sympathisent avec les indépendantistes de leur province natale, ne font pas référence au processus de décolonisation qui sous-tend des segments significatifs du mouvement indépendantiste. Les étudiants acadiens, quant à eux, semblent être uniquement tournés vers le nationalisme de leurs ancêtres. Certes, dans le discours étudiant, il y a de nombreuses références à la Déportation et à la victimisation historique des Acadiens, ${ }^{125}$ mais elles ne traduisent pas une adhésion à un projet de société alternatif. Cela émerge clairement des articles qui paraissent dans Le Reflet en 1968. En mars de cette année, l'étudiant Norbert Comeau, originaire de la baie Sainte-Marie, insiste simplement sur les droits des groupes minoritaires et la nécessité pour la majorité de se sacrifier afin de les maintenir. ${ }^{126}$ Comeau rappelle aussi à ses lecteurs que " c'est à l'école que se forme l'âme d'un peuple ", citant à l'appui Lionel Groulx, maître à penser du clérico-nationalisme du dix-neuvième et du début du vingtième siècle. ${ }^{127}$ En novembre de 1968, un autre étudiant néo-écossais, Paul D. Comeau, publie un texte analysant l'attitude des jeunes Acadiens face à leur collège, où il affirme que "c'est le[ur] devoir d'assurer l'éducation française des générations [futures] » et de "préserver l'héritage que nos ancêtres nous ont confié ". ${ }^{128}$ Le nationalisme des étudiants, comme celui de leurs aînés, se limite donc largement à la protection de la langue et ne débouche pas explicitement sur un projet politique qui irait à l'encontre du statuquo.

La vision fondamentale des étudiants francophones de Sainte-Anne n'est donc pas incompatible avec celles d'autres résidents de la région. Tous continuent à promouvoir l'idéal d'un collège bilingue qui s'inscrirait dans le rêve d'un Canada bilingue. Personne ne conteste le fait que ce bilinguisme soit à sens unique. Lorsque, en septembre 1967, l'administration du collège décide d'accueillir des étudiants anglophones, on ne leur demande pas de suivre un cheminement en français. Cela ne semble pas susciter de grandes controverses parmi les francophones. Certes, deux étudiants, J. W. Langford et David Bourque, se plaignent des insultes sournoises adressées aux unilingues Anglais. ${ }^{129}$ Sans en préciser les raisons, Bourque constate même que l'attitude face aux anglophones devient plus négative en 1968-1969, qu'en 1967-1968. Pourtant, cette hostilité est relative. Même les étudiants québécois, qui se targuent parfois de faire appel aux indépendantistes de leur province natale, se montrent beaucoup plus conciliants lorsqu'il s'agit de s'exprimer dans des 
médias anglophones. L'on n'a qu'à regarder les déclarations du soi-disant sympathisant du MIS, Jean-Pierre Vallée, qui, dans un article du Yarmouth Vanguard évoque un "bilingual college where the rights of English and French would be respected". ${ }^{130}$ L'Américain anglophone David Bourque est donc inclus dans un comité d'étudiants qui, le 11 décembre 1968, se rend à Halifax pour présenter le point de vue des grévistes au ministre d'éducation Gérald Doucet. ${ }^{131}$ Tout en prétendant vouloir garder le «Collège Sainte-Anne pour les Acadiens ", les étudiants ne s'opposent pas à la volonté du ministre d'avoir un collège qui permette aux anglophones de "développer leur langue seconde ». ${ }^{132}$ Une autre étudiante anglophone de Sainte-Anne, Gail Hersey, témoigne de la bonne entente qui y règne pendant la grève. "Before my very eyes ", écrit-elle dans Le Reflet " great unity was achieved among the French and the English; all were working for a just cause ». ${ }^{133}$ Les étudiants du Collège Sainte-Anne ne veulent pas généralement rompre la bonne-entente avec les anglophones, qui fut, depuis longtemps, au cœur de la vision de leurs aînés.

Il est intéressant que ceux qui prônent clairement la rupture avec le bon ententisme de l'Acadie néo-écossaise ne soient même pas étudiants au Collège Sainte-Anne au moment de la grève de 1968. Par exemple, c'est Cyrille LeBlanc, un Acadien originaire d'Argyle et étudiant à l'Université Moncton, qui est un des premiers à rompre avec la logique du bilinguisme institutionnel et à réclamer un réseau scolaire homogène pour les Acadiens de la Nouvelle-Écosse. ${ }^{134}$ Toutefois, pour LeBlanc, le Collège Sainte-Anne ne peut s'inscrire dans un tel réseau homogène. Avec une concentration démographique trop faible, et sans l'apport des étudiants québécois qui restent désormais chez eux suite au remplacement des collèges classiques par des Cégeps en 1967, les Acadiens du sud-ouest de la Nouvelle-Écosse ne peuvent maintenir une université homogène française. Il vaut mieux, pour LeBlanc, que les Acadiens terminent leur éducation primaire et secondaire dans des écoles unilingues françaises, puis aillent faire des études postsecondaires à l'Université de Moncton. À l'époque, les opinions de LeBlanc sont assez marginales en Nouvelle-Écosse, même parmi les étudiants qui font la grève. ${ }^{135}$ Partageant sans doute l'opinion selon laquelle Sainte-Anne ne pourrait survivre sans son caractère bilingue, les grévistes ne suggèrent jamais que le collège devienne une institution uniquement française.

En même temps, les étudiants de Sainte-Anne innovent quelque peu par rapport à leurs aînés en cherchant l'appui des Afro-Néo-Écossais. À l'automne de 1968, un groupe d'Afro-Néo-Écossais d'Halifax fondent le Black United Front (BUF), censé lutter contre la discrimination dont les Noirs étaient longtemps victimes, ${ }^{136}$ en s'inspirant, notamment, du mouvement des Black Panthers américains. Les grévistes de Sainte-Anne se montrent solidaires de la lutte des Noirs, envoyant un télégramme d'appui au BUF et affirmant, qu'à l'heure où " a second Deportation is being prepared", ils savent ce que c'est qu'être " a minority people and even worse a forgotten people ${ }^{137}$

Il reste que, malgré son association avec les Panthers, le BUF garde, du moins dans ses phases initiales, une certaine ouverture vers l'establishment néo-écossais. Sa première réunion, qui a lieu le 30 novembre 1968, est présidée par un membre du clergé : W. P. Oliver. Même si l'organisme propose de permettre aux membres de 
la communauté noire de "take charge of their own affairs and set the terms of their community's development ", ${ }^{138}$ il cherche à maintenir l'approche traditionnelle de nonconfrontation par rapport à la majorité blanche. Le Chronicle Herald salue également la fondation du BUF. ${ }^{139}$ Ainsi, même si les étudiants innovent en tendant la main à la communauté noire, cela ne peut pas non plus être considéré comme une vraie rupture idéologique avec leurs aînés.

Par ailleurs, vu que le maintien de Sainte-Anne à Pointe-de-l'Église implique non seulement des questions identitaires, mais aussi des intérêts économiques, il est facile pour les étudiants de susciter l'appui de la communauté dans son ensemble. Pendant la grève, les parents des élèves organisent des tournées des écoles secondaires du comté de Digby, où l'on cherche à mobiliser appuis supplémentaires, ou écrivent des lettres aux journaux locaux pour affirmer leur solidarité avec l'action de leurs enfants. ${ }^{140}$ Sentant l'occasion de pouvoir mettre dans l'embarras le gouvernement conservateur du premier ministre néo-écossais George I. Smith, des militants locaux du Parti libéral soutiennent activement les grévistes. ${ }^{141}$ Sous l'égide de la Chambre de commerce de Clare, l'association des "Amis du Collège Sainte-Anne " est également fondée dans le but de maintenir l'institution à Pointe-de-l'Église. Une des " amies " de premier plan est Rita Stuart, dont le magasin général dépend directement des étudiants qui fréquentent le collège. ${ }^{142}$ Enfin, probablement conscient de l'impact économique d'un déménagement pour sa communauté, le maire de Digby, Glendon Smith, accueille les grévistes et leur profere des mots d'encouragement. De leur côté, ces derniers affirment s'être présentés à la mairie afin d'obtenir des conseils du magistrat. ${ }^{143}$

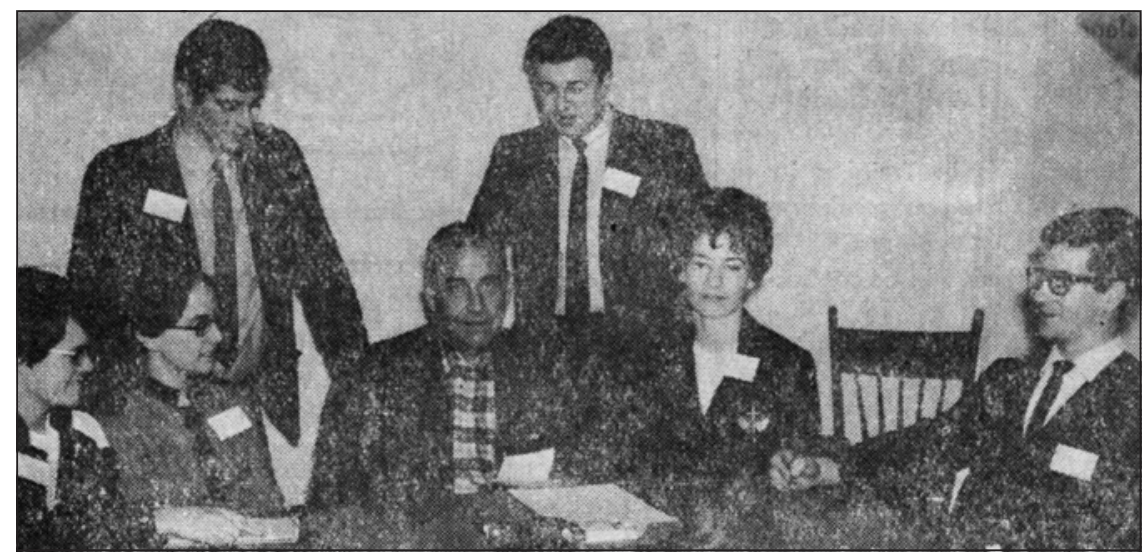

«Ste. Anne students continue strike », Digby Courier, 12 décembre 1968.

Les étudiants eux-mêmes tentent généralement de se présenter comme étant des citoyens responsables. Certes, il y a de nombreuses manifestations, ce qui fournit un visuel semblable à celui qui se répète à maintes occasions à travers l'Occident à la fin des années 1960. Toutefois, il est à noter que la communauté dans son ensemble est souvent présente lors des rassemblements et participe à leur organisation. ${ }^{144}$ 


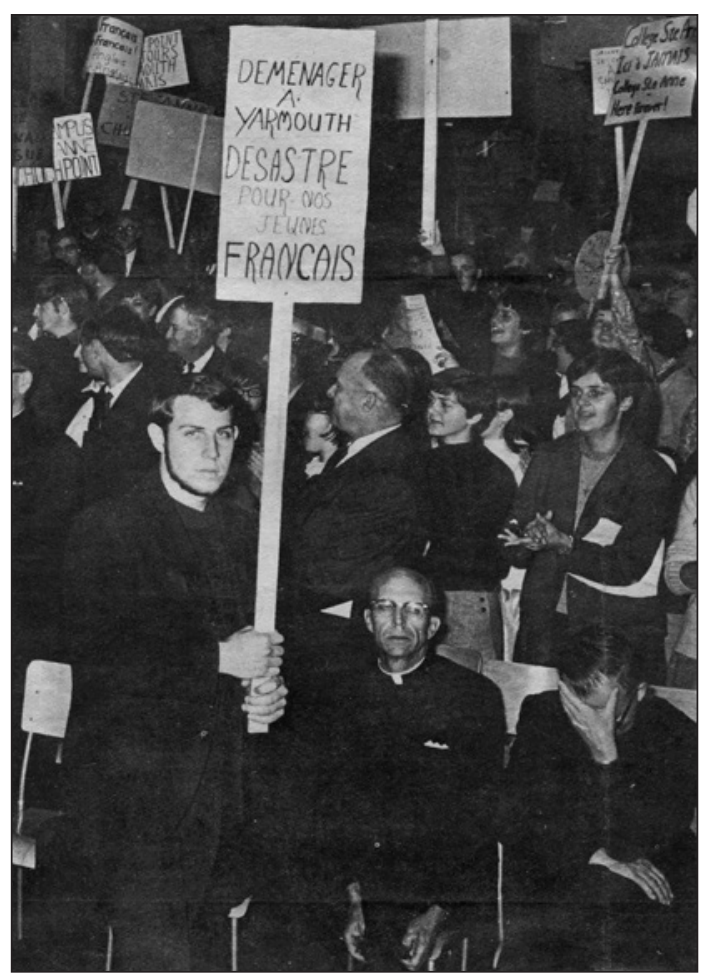

" 1500 attend college meeting ", Yarmouth Light Herald, 9 novembre 1968.

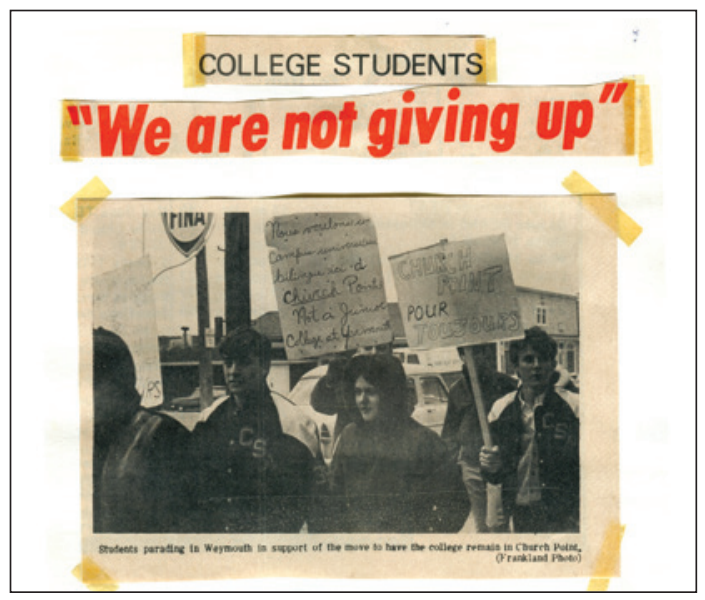

Yarmouth Vanguard, 11 décembre 1968.
Sous l'impulsion des grévistes de Sainte-Anne, les étudiants des écoles secondaires doivent même obtenir la permission écrite de leurs parents avant de pouvoir participer aux manifestations. ${ }^{145}$ En charge des relations avec les médias, l'ancien étudiant, Gérald Comeau, relate le message qu'il devait leur transmettre : «nous n'étions pas un groupe de radicals (sic), nous ne voulions pas causer des ennuis; on était là pour sauver notre université ». ${ }^{146}$

Le seul incident qui manque de "respectabilité " a lieu le 9 décembre 1968, lorsqu'un groupe d'étudiants brûlent en effigie Normand Belliveau. ${ }^{147}$ Natif de Clare et ancien étudiant du collège, Belliveau a fait une brillante carrière médicale au Québec. Membre du Grants Committee, censé établir le financement provincial des institutions postsecondaires de la province, Belliveau est aussi un ardent défenseur du déménagement vers Yarmouth. ${ }^{148}$

Cet incident, à l'instar de la livraison d'une tête de cochon au maire de Moncton, Leonard Jones, pendant la grève de février 1968, pourrait être qualifié de " charivari ». Il s'agit « d'une sorte de théâtre de rue adapté à la fonction de dénonciation publique [...]; les acteurs viennent pendant la nuit $[. .$.$] et proclament publiquement leur hostilité contre la personne visée » .^{149}$ Tout comme la tête de cochon, l'" exécution " de Belliveau suscite une certaine 


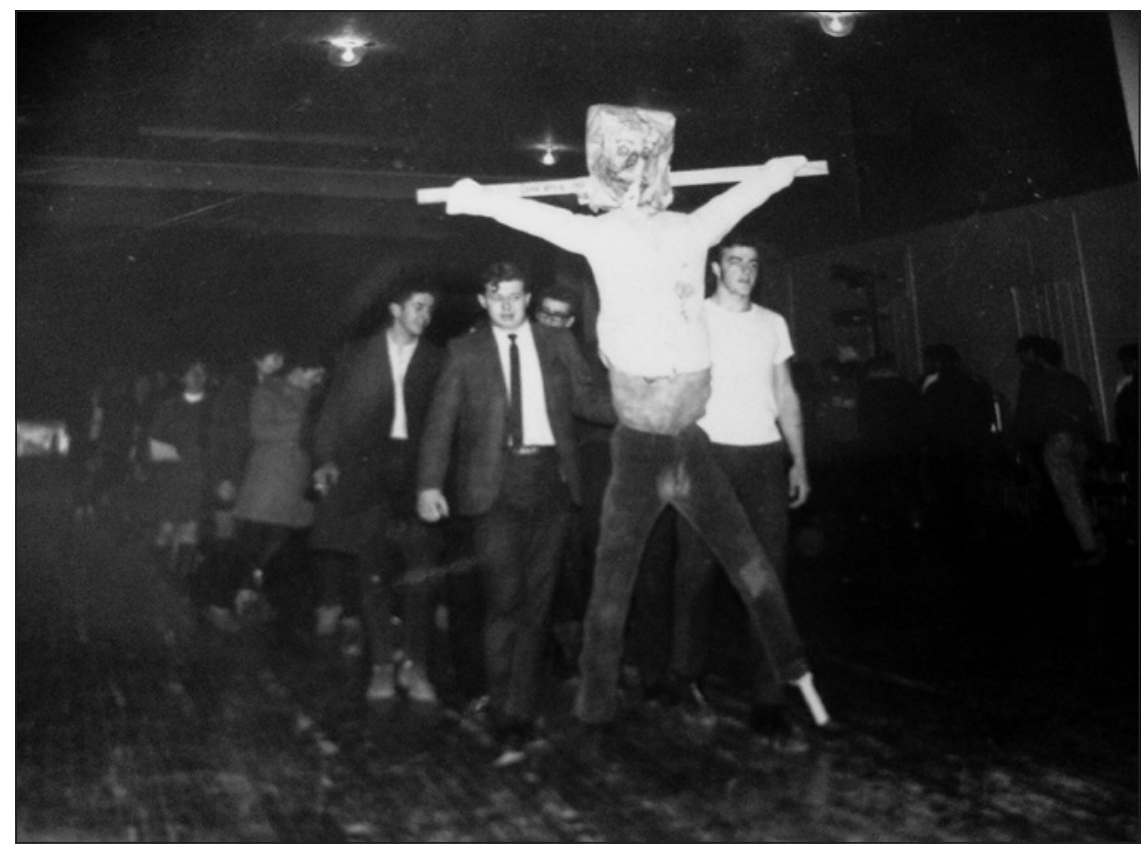

"Le docteur Belliveau brûlé en effigie ", le 9 décembre 1968. Photo de Réal Déraspe.

controverse. Malgré les rumeurs voulant que les étudiants aient été tacitement appuyés dans leur action, les professeurs et les administrateurs ne les défendent pas publiquement. ${ }^{150}$ Par ailleurs, le Chronicle Herald publie une lettre d'opinion d'une mère de famille ayant deux fils diplômés de Sainte-Anne, qui qualifie les gestes des étudiants comme étant "shameful and un-Christian-like ". ${ }^{151}$

Il reste que l'ancien gréviste Gérald Comeau prétend toujours que les étudiants ne cherchaient qu’à attirer l'attention des médias, avec un fort "visuel ». ${ }^{152}$ Selon lui, il ne faut surtout pas y voir de signe de violence. Certes, le passage du temps a peut-être affecté la manière dont Comeau présente les évènements. En revanche, il est révélateur qu'immédiatement après avoir brûlé l'effigie de Belliveau, ces mêmes étudiants exhortent les élèves de l'École supérieure de Clare ${ }^{153}$ à revenir à l'école, au lieu de continuer à boycotter leurs cours en solidarité avec leurs collègues de Sainte-Anne. ${ }^{154}$ Même à leur plus contestataire, les grévistes de Sainte-Anne font donc attention de ne pas trop bousculer les sensibilités.

Ainsi, la grève à Sainte-Anne ne représente qu'une participation timide au " moment 68 ». Bien entendu, certains étudiants se distinguent de leurs aînés en faisant appel au MIS, mais ils n'internalisent pas pleinement son idéologie. Tandis que le MIS appelle à la séparation du Québec (ou à tout le moins à des institutions homogènes françaises), les étudiants de Sainte-Anne font toujours la promotion d'un collège bilingue dans un Canada bilingue. Contrairement aux étudiants monctoniens qui, inspirés par la décolonisation, confrontent le maire Jones, ${ }^{155}$ leurs confrères de Sainte-Anne cherchent à maintenir les vieux consensus visant la bonne entente 
entre les deux communautés linguistiques de la baie Sainte-Marie. Il ne s'agit pas d'un conservatisme attribuable uniquement au statut très minoritaire des francophones en Nouvelle-Écosse; les attitudes de plusieurs étudiants anglophones (non seulement en ce qui concerne la langue, mais aussi les autres questions de société) sont tout aussi traditionalistes. Au niveau des tactiques employées, se promener avec l'effigie de Belliveau est un incident isolé et les grévistes reviennent rapidement à leur rôle de " citoyens responsables » qui appellent au retour en classe des élèves du secondaire.

\section{Conclusion}

Jusqu'à maintenant l'historiographie sur les années 1960 au Canada se concentre surtout sur les régions urbaines. La manière dont les "sixties " ont été vécues en Acadie rurale est encore assez méconnue. Avec cet article, nous avons essayé de fournir une analyse de cette période au Collège Sainte-Anne. Nous avons conclu que les étudiants de Sainte-Anne ne sont pas complètement éloignés des changements qui touchent l'Occident dans les années de l'après-guerre. Le collège se modernise, ouvrant notamment ses portes aux femmes en 1961. Rendu à la fin des années 1960, toutefois, bon nombre d'étudiants, plutôt que d'exiger une accélération des transformations, appellent au maintien du statuquo, ne se distinguant donc que très partiellement de la mentalité de leurs aînés.

Cela ne change guère avec la grève de 1968. Même si les étudiants revendiquent bruyamment le maintien du collège à Pointe-de-l'Église, appelant même à la solidarité avec des organismes comme le MIS ou le BUF, ils ne rompent jamais le pacte avec le reste de leur communauté. Appuyée généralement par l'élite politique, intellectuelle et économique, la grève de 1968 ne peut être considérée comme un appel à un monde nouveau.

Même si les étudiants de Sainte-Anne sont largement en phase avec leur communauté pendant la grève de 1968, le climat sociopolitique à Sainte-Anne évolue vraisemblablement à partir des années 1970. Les eudistes quittent définitivement le collège en 1971 et la présence de nouveaux professeurs laïcs, dont plusieurs sont formés à l'étranger, contribue sûrement à rapprocher les étudiants de Sainte-Anne de leurs confrères ailleurs en Occident. Maintiennent-ils, au moins jusqu'à un certain point, leur sensibilité conservatrice de jadis? Cette question doit faire l'objet de recherches ultérieures. Pourtant, vu que cette sensibilité était tellement ancrée, au moment même où, ailleurs en Occident, de nombreux baby-boomers contestaient violemment les structures établies, nous émettons l'hypothèse qu'il en reste des relents pour les décennies à venir. 


\section{Notes}

Nous tenons aussi à remercier Marcel Martel, Julien Massicotte, Jean-Louis Robichaud, ainsi que les évaluateurs anonymes, qui ont lu des versions antérieures du texte. Enfin, merci au personnel du Centre acadien de l'Université Sainte-Anne et du Centre d'études acadiennes de l'Université du Moncton, ainsi qu' à monsieur Réal Déraspe, un Ancien du Collège SainteAnne, pour leur aide avec les photographies.

1 François Ricard, La génération lyrique : essai sur la vie et l'œuvre des premiers-nés du babyboom (Montréal : Boréal, 1992).

2 Il s'agit du titre d'une chanson populaire des années 1970 de Stéphane Venne.

3 Cette expression est souvent utilisée pour désigner les contestations qui ont lieu dans la foulée de l'année 1968. Voir par exemple : Joel Belliveau, Le "moment 68 » et la réinvention de l'Acadie (Ottawa : Presses de l'Université d'Ottawa), 11.

4 Philippe Volpé, «La question de l'engagement de la jeunesse en Acadie du NouveauBrunswick ", HistoireEngagee.ca, 2012, http://histoireengagee.ca/la-question- delengagement-de-la-jeunesse-en-acadie-du-nouveau-brunswick/. Pour un rappel que les militants les plus bruyants ne reflètent pas nécessairement la totalité de l'opinion étudiante voir aussi ; Jean-Philippe Warren, Une douce anarchie : les années 68 au Québec (Montréal : Boréal, 2008), 252.

5 Belliveau, Le " moment 68 »; Jean-Philippe Warren et Julien Massicotte, « La fermeture du département de sociologie de l'Université de Moncton : histoire d'une crise politicoépistémologique », Canadian Historical Review 87, 3 (2006) : 463-496; Bernard Gauvin, "La question acadienne : 16 ans après les événements de 1968-1969 ", dans Khaled Belkhodja (dir.), Actes du colloque "Solidarité internationale contre l'oppression des cultures et le racisme " (Moncton : Université de Moncton, 1986), 81-90; Barbara LeBlanc, "Tête à tête et charivari à Moncton : rencontre interculturelle entre les Acadiens et les anglophones de Moncton ", Les Cahiers de la Société historique acadienne 27, 1 (1996) : 4-18; Raymond G. LeBlanc, La contestation étudiante, thèse de maîtrise (philosophie), Université de Moncton, 1970; Lise Ouellette, Les luttes étudiantes à l'Université de Moncton : production ou reproduction de la société acadienne?, thèse de maîtrise (sociologie), Université de Montréal, 1982.

6 Peter Kent, Inventing Academic Freedom: The 1968 Strax Affair at the University of New Brunswick (Halifax, Formac, 2012).

7 Ian Milligan, Rebel Youth : 1960s Labour Unrest, Young Workers and New Leftists in English Canada (Vancouver : UBC Press, 2014), 136-147.

8 James D. Cameron, For the People: A History of St-Francis Xavier University (Montréal et Kingston : McGill-Queens University Press, 1996), 375.

9 René LeBlanc et Micheline Laliberté, Sainte-Anne, collège et université, 1890-1990 (Pointe-de-l'Église : Chaire d'étude en civilisation acadienne de la Nouvelle-Écosse, Université Sainte-Anne, 1990), 345-347.

10 Par exemple, aucun des récents ouvrages récents analysés dans le bilan historiographique tracé par Philippe Volpé n'aborde l'Acadie de la Nouvelle-Écosse. Voir : Philippe Volpé, "Autour de l'éducation : plaidoyer pour une histoire incarnée de l'Acadie ", Acadiensis XLIV, 2 (2015) : 131-148.

11 Nous empruntons ce terme à Pierre Bourdieu qui le définit comme " un système de valeurs [...] à partir duquel [les gens] engendrent des réponses à des problèmes extrêmement différents ». Voir : Pierre Bourdieu, Questions de sociologie (Paris : Éditions de Minuit, 1984), 228.

12 Ricard, La génération lyrique, 7-13. Voir aussi Doug Owram, Born at the Right Time : A History of the Baby Boom Generation (Toronto : University of Toronto Press, 1996), xiii-xiv. 
13 Pensons notamment à l'expansion de l'État, l'évolution de la foi religieuse, la prospérité économique, et l'influence du processus de décolonisation. Ces thèmes sont développés dans la section "L'esprit des sixties".

14 Entrevue de Megan Blanchard avec Nicole Muise, 2015, 29 : 13.

15 Entrevue de Mylène Comeau avec Guy LeBlanc, 2015, 14 :58

16 Joel Belliveau arrive à la même conclusion dans son étude de la grève à Moncton, voir Belliveau, Le "moment 68 ", 26.

17 La municipalité de Clare est aussi souvent identifiée comme la région de « la baie Sainte-Marie ». Avec une population d'environ neuf mille habitants, elle est située dans le comté de Digby, à la frontière du comté de Yarmouth.

18 "Historique de la querelle du déménagement de Sainte-Anne ", L'Évangéline, 20 décembre $1968: 2$.

19 Pour une liste complète d'étudiants voir " Annuaire 1968-169 », Centre acadien (CA) Fonds Université Sainte-Anne, Boîte 9, Dossier 78.

20 Belliveau Le «moment 68 », 188.

21 Ibid., 282.

22 Ibid., 28.

23 Quelques numéros sont conservés au Centre acadien de l'Université Sainte-Anne, mais nous avons eu accès à la collection exhaustive de monsieur Réal Déraspe, étudiant au moment de la grève de 1968.

24 Inauguré en 1963, Le Reflet est un mensuel dont la publication n'est pas toujours régulière. Il n'est plus publié à partir de 1996.

25 À partir de novembre 1967, les activités de l'association étudiante du Collège SainteAnne ne sont pas non plus encadrées par le corps professoral. Voir Alain Deveau, "Prise de conscience de l'AGECSA », Le Reflet, $1^{\text {er }}$ décembre $1967: 1$.

26 Clarence J. D’Entremont, "Opinion du lecteur », Le Reflet, 14 mars 1968 : 6. Mis à part une menace de contacter le Père supérieur au sujet des " ordures " publiées dans Le Reflet, D'Entremont n'explique pas ce qui l'a précisément offusqué dans le dernier numéro du journal.

27 Les archives de l'Association étudiante du Collège Sainte-Anne n'ont malheureusement pas été conservées.

28 Il s'agit de Nicole Muise (Boudreau), Réal Déraspe (entrevue non enregistrée), Madeleine Deveau, Jean-Pierre Gaudet, Gail Hersey, Guy LeBlanc et André Turbide. Nous avons aussi écouté l'entrevue accordée par Gérald Comeau, dans le cadre du $125^{\mathrm{e}}$ anniversaire de l'Université Sainte-Anne. Voir : Université Sainte-Anne, 125 années d'histoire(s) à raconter: Manifestations étudiantes, https://www.usainteanne. $\mathrm{ca} / 125$-manifestations-etudiantes. Malheureusement, plusieurs autres étudiants qui ont participé activement à la grève de décembre 1968 sont maintenant décédés. D’autres n'étaient pas joignables. Malgré les lacunes, il s'agit quand même d'un échantillon assez diversifié : une anglophone de la Nouvelle-Écosse, trois Québécois et quatre Acadiens de la baie Sainte-Marie.

29 Pour une critique influente de la pratique de l'histoire orale, voir Patrick O'Farrell, "Oral History : Facts and Fiction ", Quadrant 23, 11 (1979) : 4-9.

30 Stuart Henderson, Making the Scene : Yorkville and Hip Toronto in the Sixties (Toronto: University of Toronto Press, 2008), représente un modèle particulièrement utile. Voir aussi Catherine Foisy et Steven High, «Un chantier à réinvestir ou à réinventer... : histoire contemporaine du Québec et sources orales ", Revue d'histoire de l'Amérique française 69, 1-2 (2015) : 11-18. Pour un bon survol des débats méthodologiques actuels en histoire orale, voir Andréanne LeBrun, « Retour sur la journée d'étude "L'histoire orale en vaut-elle la peine? Avantage et contributions d'une pratique en milieu francophone" ", Bulletin de liaison des adhérents de l'AFAS, http://afas.revues. org/2953; Robert Perks et Alastair Thomson (dir.), The Oral History Reader (New-York : Routledge, 2015). 
31 Jacques Paul Couturier, Construire un savoir : l'enseignement supérieur au Madawaska, 1946-1974 (Moncton, Éditions d'Acadie, 1999), 189, 197-198.

32 Nicolas Landry, Histoire de l'enseignement universitaire à Shippagan (Québec : Éditions de la francophonie, 2012), 118-119, 125-126.

33 Nicolas Landry, Un collège classique en Acadie du Nouveau-Brunswick : le Sacré-Coeur de Caraquet_Bathurst, 1899-1975 (Québec : Éditions de la Francophonie, 2014).

34 Couturier, Construire un savoir, 198.

35 Landry, Un collège classique 280.

36 Ibid., 208-213.

37 Ibid., 284.

38 Ibid., 8.

39 LeBlanc et Laliberté, Sainte-Anne.

40 Eloi Vigneault, «Un renouveau nécessaire ", Le Reflet, $1^{\text {er }}$ décembre $1967: 2$; « SteAnne's Students Support Black Front ", Halifax Chronicle Herald, 11 décembre 1968 : 8; Denis Bradet, "Problème à l'échelle nationale ", Le Reflet, 7 novembre 1968: 2.

41 Fondé en novembre 1968, suite à une visite des Black Panthers à Halifax, le Black United Front vise à promouvoir "l'auto-détermination des Noirs » en Nouvelle-Écosse. Le Mouvement pour l'intégration scolaire, également fondé en 1968, lutte pour un réseau scolaire unilingue français et, ultimement, pour l'indépendance du Québec. Voir J. S. W. Walker, "Black Confrontation in Sixties Halifax ", dans Lara Campbell et Dominique Clément (dirs.), Debating Dissent: Canada and the 1960s (Toronto : University of Toronto Press, 2012), 173-193; Sean Mills, The Empire Within : Postcolonial Thought and Political Activism in Sixties Montreal (Montreal-Kingston : McGill-Queen's University Press, 2010), 142.

42 Owram, Born at the Right Time, 218; James Pitsula, As One Who Serves: The Making of the University of Regina (Montreal et Kingston : McGill-Queen's University Press, 2006), 5.

43 Owram, Born at the Right Time, 306; Milligan, Rebel Youth, 9.

44 Belliveau, Le "moment 68 ", 47.

45 Voir par exemple, Belliveau, Le "moment 68 ", 183; James, Pitsula, New World Dawning: The Sixties at Regina Campus (Regina : Canadian Plains Research Center, 2008), 249.

46 Patricia Jasen, " "In Pursuit of Human Values (or Laugh When You Say That)" The Student Critique of the Arts Curriculum in the 1960s ", dans Paul Axelrod and John G. Reid (dirs.), Youth, University, and Canadian Society : Essays in the Social History of Higher Education (Montreal et Kingston : McGill-Queen's University Press, 1989), 249.

47 Owram, Born at the Right Time, 274-275.

48 Belliveau, Le " moment 68 ", 101.

49 Selon plusieurs chercheurs, l'origine de ces changements au Canada français peut être retracée au renouveau du catholicisme dans les années 1930. Voir Jean-Philippe Warren et E. Martin Meunier, Sortir de la " Grande noirceur ". L'horizon "personnaliste " de la révolution tranquille (Québec, Septentrion, 2002); Michael Gauvreau, The Catholic Origins of Quebec's Quiet Revolution 1931-1970 (Montréal et Kingston : McGillQueens University Press, 2005). En Acadie, Julien Massicotte explore aussi l'influence de la pensée personnaliste sur le père Clément Cormier, un des fondateurs de l'École des sciences sociales au Collège Saint-Joseph de Memramcook en 1939. Voir Julien Massicotte, "Portrait d'un "fondateur dans l'âme" : Clément Cormier, pionnier des sciences sociales en Acadie du Nouveau-Brunswick ", Acadiensis XXXVIII, 1 (2009) : 3-32; Julien Massicotte, "La Question d'une tradition sociologique en Acadie : un aperçu ", Canadian Review of Sociology 45, 3 : 267-304. 
50 Ricard, La génération lyrique, 76. La manifestation de ce phénomène est bien décrite dans Landry, Un collège classique en Acadie du Nouveau-Brunswick, 141-177.

51 Louise Bienvenue, Ollivier Hubert et Christine Hudon, Le collège classique pour garçons, études historiques sur une institution québécoise disparue (Montréal : Fides, 2014), 299300. Sur le même sujet voir Simone LeBlanc-Rainville, Corinne Gallant : une pionnière du féminisme en Acadie (Moncton : Institut d'études acadiennes, 2012).

52 Michel Bock, "L'Association de la jeunesse franco-ontarienne (1949-1972) : témoin et actrice d'une mutation idéologique ", dans Michel Bock (dir.), La jeunesse au Canada français : formation, mouvements et identité (Ottawa : Presses de l'Université d'Ottawa, 2007), 206; cité dans Landry, Un collège classique en Acadie du Nouveau-Brunswick, 252.

53 Belliveau, Le "moment 68 ", 83, 105; Landry, Un collège classique en Acadie du Nouveau-Brunswick, 252-254.

54 Ricard, La génération lyrique, 78.

55 Warren, Une douce anarchie, 242.

56 Joel Belliveau et Frédéric Boily, « Deux révolutions tranquilles? Expériences néobrunswickoise et québécoise comparées ", Recherches sociographiques 46, 1 (2005): 11-34; Della Stanley "The 1960s : The Illusions and Realities of Progress ", dans E.R. Forbes et D. A. Muise (dirs.), The Atlantic Provinces in Confederation (Toronto : University of Toronto Press, 1991), 439, 442; Walker «Black Confrontation », 179.

57 Au Nouveau-Brunswick, les hommes politiques acadiens comme Louis Robichaud, premier ministre de 1960 à 1970, sont au cœur de cette expansion de l'État. Voir Belliveau et Boily " Deux révolutions tranquilles... ». En Nouvelle-Écosse, les Acadiens, notamment ceux de Clare, sont également présents sur la scène politique. Depuis 1838, jusqu'à l'abolition de la circonscription en 2012, ils ont toujours été représentés par un Acadien à l'Assemblée législative leur donnant « a political strength and a recognition which none of the other Acadian regions have been able to attain ». Voir Sally Ross \& Alphonse Deveau, The Acadians of Nova Scotia. Past and Present (Halifax : Nimbus, 1992), 162.

58 Africville est un quartier Afro-néo-écossais de Halifax, dont les habitants sont expropriés entre 1964 et 1969. Pour un survol de la désillusion de la fin des années 1960 et du début des années 1970, voir Stanley, "1960s »; John Reid, «The 1970s. Sharpening the Sceptical Edge ", dans E. R. Forbes et D. A. Muise (dirs.), The Atlantic Provinces in Confederation.

59 Belliveau, Le " moment 68 ", 182.

60 Jean-Philippe Warren et Andrée Fortin, Pratiques et discours de la contreculture au Québec (Québec : Septentrion, 2015), 13.

61 Marcel Martel, "They smell bad, have diseases and are lazy" : RCMP Officers Reporting on Hippies in the Late Sixties ", Canadian Historical Review 90, 2 (2009) : 215-245.

62 Mills, The Empire Within; Jean-Philippe Warren et Julien Massicotte, «La fermeture du département de sociologie de l'Université de Moncton : histoire d'une crise politico-épistémologique ", Canadian Historical Review 87, 3 (2006) : 463-496; Tudi Kernalegenn, "Le réveil des revendications régionalistes et nationalitaires au tournant des années 1968 : analyse d'une "vague" nationale », Fédéralisme-Régionalisme 13, 1 (2013).

63 Patrick Webber, " "For a Socialist New Brunswick" : The New Brunswick Waffle, 1967-1972 ", Acadiensis XXXVIII, 1 (2009) : 75-104; Milligan, Rebel Youth, 140.

64 Michelle Landry, « Pour en finir avec le passage à la modernité des francophonies canadiennes ", dans Michelle Landry, Martin Pâquet, and Anne Gilbert (dirs.), Mémoires et mobilisations (Québec : Presses de l'Université Laval, 2015), 229.

65 Pour une explication de "modernité " versus " modernisation " voir Landry " Pour en finir... ", 235; Jean-Philippe Warren " Petite typologie philologique du "moderne" au 
Québec (1850-1950). Moderne, modernisation, modernisme, modernité ", Recherches sociographiques 46, 3, (2005) : 495-525. Voir aussi Julien Massicotte, L’Acadie du progrès et du désenchantement 1960-1994, thèse de doctorat en histoire, Université Laval, 2011, 36-37.

66 Mentionnons, surtout, une plus grande présence du catholicisme, une culture politique rigidement bipartite (libéralo-conservatrice), ainsi qu'une économie encore très dépendante des ressources primaires. Marc-Adélard Tremblay, «Les Acadiens de la Baie française. L'histoire d'une survivance ", Revue d'histoire de l'Amérique française 15, 4 (1962) : 550; Alphonse Deveau, Clare. La ville française. Tome II (Pointe-de-l'Église : Les historiens d'âge d'or de la Baie Sainte-Marie, 1982).

67 Tremblay interprète ces changements comme reflétant le passage entre un mode de vie traditionnel et moderne. Des travaux ultérieurs servent à nuancer cette interprétation. Voir Landry, " Pour en finir... ", 223.

68 Tremblay, "Les Acadiens de la Baie française " : 535-543.

69 Tremblay, "Les Acadiens de la Baie française " : 540; Ministère de l'Industrie et du Commerce, Recensement de 1961, Population : langue maternelle comtés et subdivisions, 9.

70 Alphonse Deveau, Clare. La ville française, 440.

71 Tremblay «Les Acadiens de la Baie française » 552.

72 Marc-Adélard Tremblay, "La société acadienne en devenir : l'impact de la technique sur la structure sociale globale ", Anthropologica 8, 2 (1966) : 8, 11-14.

73 LeBlanc et Laliberté, Sainte-Anne, 322-323.

74 Ibid., 246.

75 Ibid., 417.

76 Ibid., 281; Correspondance avec Jean-Louis Robichaud, juillet 2015.

77 Robert Gaudet, "Critique positive ", Le Reflet, $1^{\text {er }}$ décembre 1967 : 1. Dans son texte, Gaudet rappelle le fait que " quand nous avons critiqué, nous avons critiqué positivement [ainsi] nous sommes déjà considérés comme des adultes et nous agissons ainsi ".

78 Alain Deveau, "Prise de conscience de l'AGECSA », Le Reflet, $1^{\text {er }}$ décembre 1967 : 1 ; Gaudet, « Critique positive » 1.

79 Eloi Vigneault, "Un renouveau nécessaire ", Le Reflet, $1^{\text {er }}$ décembre 1967, : 2; Landry, Un collège classique en Acadie du Nouveau-Brunswick, 254.

80 Vigneault, "Un renouveau nécessaire ».

81 Ernest Buckler, "This is no Piffling Backwoods ", The Star Weekly, 6 octobre 1965: 16-18; LeBlanc et Laliberté, Sainte-Anne, 323-324.

82 LeBlanc et Laliberté, Sainte-Anne, 315.

83 "Report of the University Grants Committee for the Year Ending December 31 1966 ", cité dans LeBlanc et Laliberté, Sainte-Anne, 327.

84 D'où l'accueil chaleureux fait au premier ministre néo-brunswickois Louis Robichaud lors de sa visite au Collège Sainte-Anne dans le cadre de la Convention nationale acadienne de 1960. LeBlanc et Laliberté, Sainte-Anne, 319.Voir aussi Tremblay, «La société acadienne en devenir » : 11-12, 21-23.

85 Ministère de l'Industrie et du Commerce du Canada, Recensement de 1961, 9-10.

86 "Clare Issues Strong Protest Against Ste-Anne's College Moving to Yarmouth ", Yarmouth Light Herald, 10 novembre, 1966 : 1-2. Le désir des élites professionnelles acadiennes de la baie Sainte-Marie de maintenir des relations cordiales avec la communauté anglophone pendant les années 1960 est évident dans d'autres articles journalistiques de l'époque. Voir Buckler, "This is no Piffling Backwoods » : 16-18.

87 Selon le recensement de 1961, 16 \% des résidents de Clare sont anglophones.

88 LeBlanc et Laliberté, Sainte-Anne, 337.

89 Ibid., 343-344.

90 Ibid., 354. 
91 "Les élèves du Collège Sainte-Anne se sont mis en grève hier matin ", L'Évangéline, 6 décembre $1968: 1$.

92 Alban D’Entremont « La grève....avant, pendant, après ", Le Reflet, 24 janvier 1969 : 1.

93 "Communiqué de presse, 6 décembre 1968 "; "Lettre d'Onil Dionne à Mgr Austin Burke, le 5 décembre 1968 »Centre acadien (CA) Fonds Université Sainte-Anne, Boîte 38, Dossier : Le futur du Collège.

94 LeBlanc et Laliberté, Sainte-Anne, 355-356.

95 Le Collège Sainte-Anne est renommé «l'Université Sainte-Anne » en 1977.

96 La volonté des étudiants monctoniens de dépasser la question du financement de l'université pour s'inscrire dans un "vaste mouvement contestataire occidental » est bien notée par plusieurs acteurs de l'époque. Voir Belliveau «Le moment 68 », 17-18.

97 Michael Oakeshott, Rationality in Politics (London : Methuen, 1962).

98 Danic Parenteau et Ian Parenteau, Les idéologies politiques (Québec : Presses de l'Université du Québec, 2008), 67-68.

99 Alain Deveau, «Prise de conscience de l'AGECSA », Le Reflet, $1^{\text {er }}$ décembre $1967: 1$.

100 Jean-Robert Turcotte, "Éditorial ", Le Reflet, 7 novembre $1968: 2$.

101 Madeleine Deveau, "Changes in Our Midst ", Le Reflet, $1^{\text {er }}$ décembre $1967: 4$.

102 Robert Gaudet, "Mariage à l'essai », Le Reflet, 18 avril 1968 : 1.

103 Anna Marie Thibodeau, "The Glory of Motherhood ", Le Reflet, $1^{\text {er }}$ décembre $1967: 4$.

104 David Bourque, "Our world", Le Reflet, 24 janvier 1969 : 1; David Bourque "Would I Fight if Called? ", Le Reflet, $1^{\text {er }}$ mars $1969: 3$. Bourque retournera aux États-Unis après la fin de ses études à Sainte-Anne.

105 Bourque est également élu président de l'association étudiante du collège en février 1969. Voir " Nouvel exécutif pour l'A.G.E. ", Le Reflet, $1^{\text {er }}$ mars 1969: 1.

106 Barbara Erjavec, "My Daughter was on Strike for Sainte Anne », Digby Courier, 19 décembre 1968, 2e section : 1 .

107 Entrevue de Daniel Rogers avec Gail Hersey, 2015, $6: 30-6: 49$.

108 Entrevue de Mylène Comeau avec Madeleine Deveau, 2015, 5 : 40-6 : 30. De tels désaccords entourant le code vestimentaire se font aussi sentir au Collège Maillet à la même époque. Voir Couturier, Construire un savoir, 189.

109 Entrevue de Mylène Comeau avec Madeleine Deveau, 2015, 5 :00-5:07

110 J. W. Langford, "Changes (?) St-Anne », Le Reflet, 28 mars 1969 : 1; David Bourque, "Black-balled ", 7 novembre $1968: 1$.

111 Dans d'autres contextes, la « mentalité minoritaire » de certains militants acadiens aurait modéré leurs revendications. Voir Michael Poplyansky, «Unilinguisme québécois et bilinguisme acadien : les politiques linguistiques du Parti québécois et du Parti acadien, 1970-1978 ", Francophonies d'Amérique 34 (2012) : 155-171.

112 Ferdinand Tönnies et Charles P. Loomis (trad.), Community \& Society (New-York, Harper \& Row, 1963), 43.

113 Peter Kent, Donald Savoie et Ian Milligan notent la participation active d'individus venant de l'extérieur de la communauté locale dans les contestations étudiantes à la University of New-Brunswick, l'Université de Moncton et au King's College. Voir Kent, Inventing Academic Freedom, 100; Donald J. Savoie, Moi, je suis de Bouctouche (Montréal-Kingston : McGill-Queen's University Press, 2009), 112; Milligan, Rebel Youth, 140. Pour un bref examen des liens entre les étudiants de ces institutions et leurs collègues ailleurs au Canada, voir Belliveau, "Le moment 68 ", 103-104; Kent, Inventing Academic Freedom, 60-61. Même si le Collège Sainte-Anne est représenté lors de la fondation du Nova Scotia Union of Students en 1967, Le Reflet ne révèle pas de grande participation aux instances étudiantes pancanadiennes.

114 Kent, Inventing Academic Freedom; Warren et Massicotte, « La fermeture du département de sociologie de l'Université de Moncton ». Voir aussi les témoignages de Roger Savoie et Alain Even où ils relatent, à la fois leur rapport tendu avec la 
communauté acadienne de Moncton, et l'intérêt des étudiants pour leurs idées. Roger Savoie, "Mes années 60 en Acadie ", HistoireEngagee.ca, 2015, http://histoireengagee. $\mathrm{ca} / \mathrm{mes}$-annees-60-en-acadie/; Adeline Vasquez-Parra, " "68 et nous" : une entrevue avec le sociologue Alain Even ", HistoireEngagee.ca, 2012, http://histoireengagee.ca/ lactualite-en-debat-68-et-nousune-entrevue-avec-le-sociologue-alain-even/\#more-17402

115 Euclide Chiasson est particulièrement vigoureux dans sa critique des organisations traditionnelles acadiennes, comme la Société nationale des Acadiens, qui négligent, selon lui, la réalité socio-économique du nord-est du Nouveau-Brunswick. Voir Michael Poplyansky, "Acadien ou francophone? Indécision identitaire au NouveauBrunswick et en Nouvelle-Écosse 1968-1973 ", Port Acadie : Revue interdisciplinaire en études acadiennes 27 (2015) : 63-81. Notons, cependant, que Chiasson ne cesse de rappeler que son action constitue un retour à " un paradis perdu ", et non un " émerveillement naïf pour tout ce qui est neuf ». À sa façon, il cherche aussi à maintenir une certaine continuité par rapport aux aînés. Voir Euclide Chiasson et al., Le Parti Acadien (Montréal : Parti pris, 1972), 40.

116 Entrevue de Daniel Rogers avec Gail Hersey, 2015, 2 :46. Il est à noter que l'année 1968-1969 est marquée par l'arrivée de plusieurs nouveaux professeurs laïcs, formés à l'étranger. Certains d'entre eux demeureront à Sainte-Anne pour plusieurs décennies. Il reste à savoir à quel point ce renouvellement du corps professoral transforme la culture étudiante de Sainte-Anne au cours des années 1970.

117 Olivette D’Entremont, "Visite à Moncton ", Le Reflet, 14 mars 1968 : 3. Pour un bref résumé de la pensée personnaliste, voir Meunier et Warren, Sortir de la " Grande noirceur", 69.

118 "Les francophones de Church Point font la grève pour conserver l'unique collège bilingue de la Nouvelle-Écosse ", Le Soleil, 7 décembre 1968, CA, Fonds Université Sainte-Anne, Boîte 43, Dossier 316. Pour une histoire du MIS et une analyse de l'impact de la décolonisation sur ce regroupement voir Mills, The Empire Within, 142.

119 "Les francophones de Church Point font la grève pour conserver l'unique collège bilingue de la Nouvelle-Écosse » Le Soleil, 7 décembre 1968, CA Fonds Université Sainte-Anne, Boîte 43, Dossier 316.

120 Denis Bradet, "Problème à l'échelle nationale ", Le Reflet, 7 novembre $1968: 2$.

121 Mark Bradley, "Opinion du lecteur », Le Reflet, 14 mars 1968 : 6-7.

122 "Student will Continue Strike, Press Minister for Decision ", Halifax Chronicle Herald, 9 décembre $1968: 17$.

123 " Lettre de Jean-Marie Nadeau à Gérald Doucet, le 10 décembre 1968 », Société historique Nicolas-Denys, Fonds Collège de Bathurst, Dossier : Correspondance 1967-1968.

124 Les étudiants de Sainte-Anne n'adoptent pas non plus l'idéologie d'extrême gauche qui anime certains de leurs confrères des universités anglophones. Pour une analyse de la participation d'étudiants du King's College à la "lutte ouvrière " au tournant des années 1970, voir Milligan, Rebel Youth, 136-147.

125 "Ste-Anne's Students Support Black Front ", Halifax Chronicle Herald, 11 décembre 1968 : 8; "Placards and Faces Join the St. Anne College Auditorium ", La voix de Clare, le 6 novembre $1968: 2$.

126 Norbert Comeau, "Le dilemme du site ", Le Reflet, 14 mars $1968: 4$.

127 Pour une fine analyse de la pensée de Groulx, notamment en lien avec les francophonies minoritaires, voir Michel Bock, Quand la nation débordait les frontières : les minorités françaises dans la pensée de Lionel Groulx (Montréal : Hurtubise HMH, 2004).

128 Paul D. Comeau, "L'attitude des jeunes Acadiens ", Le Reflet, 7 novembre $1968: 3$.

129 J.W. Langford, "College Ste-Anne-Bilingual? » Le Reflet, 24 janvier 1969 : 6; David Bourque, "Bilingualism " Le Reflet, 10 octobre 1968: 2 .

130 "Committee recommends College move ", Yarmouth Vanguard, 6 novembre 1968 : $1-2$. 
131 "Education Minister to Meet Today with Student Protesters", Halifax Chronicle Herald, 11 décembre $1968: 13$.

132 «Rencontre avec M. Doucet, 11 décembre 1968 », Centre acadien (CA) Fonds Université Sainte-Anne, Boîte 38, Dossier : Le futur du Collège.

133 Gail Hersey, "Views Towards C.S.A. Strike ", Le Reflet, 24 janvier 1969 : 2.

134 Nick Fillmore, "Acadians Facing Identity Crisis ", The $4^{\text {th }}$ estate 1, 7 (1969) : 1, 13.

135 Correspondance avec Jean-Louis Robichaud, juillet 2015. Voir aussi Sally Ross, Les écoles acadiennes en Nouvelle-Écosse (Moncton, Centre d'études acadiennes, Université de Moncton, 2001), 100-106.

136 Walker, "Black Confrontation in Sixties Halifax », 173-193.

137 "Ste-Anne’s Students Support Black Front », Halifax Chronicle Herald, 11 décembre $1968: 8$.

138 Walker, «Black Confrontation », 175.

139 Ibid., 189.

140 "College Ste. Anne Students get New Support in Protest Strike ", Halifax Chronicle Herald, 7 décembre $1968: 11$; Barbara Erjavec, « My Daughter» $: 1$.

141 Entrevue de Mylène Comeau avec Guy LeBlanc, 2015, 6 :28

142 "Les amis du Collège ", Petit Courier de la Nouvelle-Écosse, 26 décembre $1968: 4$.

143 «Ste. Anne Students Continue Strike ", Digby Courier, 12 décembre 1968 : 1.

144 "College Ste. Anne Students get new Support in Protest Strike » Halifax Chronicle Herald, 7 décembre 1968 : 11; "Le diocèse de Yarmouth préconise une aide financière adéquate pour le Collège Sainte-Anne ", L'Évangéline, 7 décembre $1968: 1$.

145 Entrevue de Mylène Comeau avec Guy LeBlanc, 2015, 4 :30-4 :35.

146 Voir l'entrevue de Gérald Comeau, Université Sainte-Anne, 125 années d'histoire(s) à raconter: Manifestations étudiantes, https://www.usainteanne. $\mathrm{ca} / 125$-manifestations-etudiantes.

147 "Le Dr. Belliveau brûlé en effigie ", L'Évangéline, 10 décembre, 1968 : 1.

148 LeBlanc et Laliberté, Sainte-Anne, 351.

149 LeBlanc, "Tête à tête et charivari à Moncton " : 12. Pour une analyse du carnaval et du charivari comme moyens séculaires de contestation en Acadie, voir Denis Bourque et Anne Brown (dirs.), Littératures d'expression française d'Amérique du Nord et le carnavalesque (Moncton, Éditions d'Acadie, 1998), 13.

150 LeBlanc et Laliberté, Sainte-Anne, 354.

151 Estelle D'Éon, " Remain where it is ", Halifax Chronicle Herald, 26 décembre 1968 : 4.

152 Voir l'entrevue de Gérald Comeau, Université Sainte-Anne, 125 années d'histoire(s) à raconter: Manifestations étudiantes, https://www.usainteanne.ca/125-manifestationsetudiantes. Il est intéressant que les étudiants décident de brûler l'effigie de Belliveau, un "Acadien de Montréal " plutôt que celle d'un résident bien en vue d'Argyle, tel le père Clarence D'Entremont, qui appuyait aussi le déménagement. Un tel geste aurait sûrement provoqué un émoi encore plus grand au sein de la communauté. Voir Maurice Rainville et Simone LeBlanc-Rainville, Le rassembleur. Léger Comeau (Moncton : Éditions d'Acadie, 2000), 124.

153 Il s'agit de l'ancien nom de l'École secondaire de Clare. Les étudiants commencent à boycotter leurs cours dès le 6 décembre 1968 en geste de solidarité. "Students of College Ste-Anne go on strike ", Halifax Chronicle Herald, 6 décembre 1968: 25.

154 «Le Dr. Belliveau brûlé en effigie ", L'Évangéline, 10 décembre, 1968 : 1.

155 Belliveau, "Le moment 68 », 228. 